\title{
Estimation of the Population of Stars in the Universe
}

\author{
Ignacio Pablo Traversa-Tejero \\ Department of Natural Sciences, Northern Regional Teachers Center, Rivera, Uruguay \\ Email: igtraversa@gmail.com
}

How to cite this paper: Traversa-Tejero, I.P. (2021) Estimation of the Population of Stars in the Universe. Open Access Library Journal, 8: e8154.

https://doi.org/10.4236/oalib.1108154

Received: November 5, 2021

Accepted: December 25, 2021

Published: December 28, 2021

Copyright ( $) 2021$ by author(s) and Open Access Library Inc.

This work is licensed under the Creative Commons Attribution International License (CC BY 4.0).

http://creativecommons.org/licenses/by/4.0/

\section{(c) (i) Open Access}

\begin{abstract}
Stars are light years away, and their brightness depends on multiple variables. Starting in the 20th century, artificial satellites were launched that added to the bright bodies of the observable sky. From 26.816 satellite observations and 9.037 stars visible to the naked eye, regression models were made between brightness and distances. The existence of a visual convergence plane that receives light photons arriving with a delay, $c=299.792 .458 \mathrm{~m} \cdot \mathrm{s}^{-1}$ was assumed. Under the principle of large-scale homogeneity and isotropy, the objective of the study was to develop models to estimate the population of stars in the universe. It is concluded that the correlation between the satellite brightness model and the surface light quantity model presented a high adjustment, so that the value of the a priori probability of emitting sources of photons (stars) per surface derives from the probability gradient theorem (TGP). The value $4.62 \mathrm{E}-9$ starlight $\mathrm{m}^{-2} \cdot \mathrm{s}^{1}$, is a universal constant obtained from the delay (speed) of light. The velocity of wave-bodies is associated with their probability of occurrence. The probability of physical phenomena is regulated by randomness which explains $50 \%$ and by causality which explains the remaining $50 \%$. Light has a probability that arises from its speed, from that probability it is estimated that the population of stars in the universe is $4.5 \mathrm{E} 24$.
\end{abstract}

\section{Subject Areas}

Astrophysics, Cosmology, Theoretical Physics

\section{Keywords}

Probability Gradient Theorem, Visual Convergence Plane, Total Star Population

\section{Introduction}

The human eye can distinguish objects up to a certain size as long as they are 
contrasted with the background where they are located. The principle of muscular accommodation of optics proposes a reflex reaction of the eyes (Raasveldt, 1958) [1] and although the observation of man is three-dimensional, the visual perception of the sky with the naked eye adapts to a two-dimensional field (García et al., 1997 [2]; Storchi-Bergmann, 2012 [3]). Also, stellar brightness is an apparent or relative magnitude, so higher magnitude stars may appear less bright because they are farther from Earth than stars that appear brighter because they are closer.

In ancient times, the Greek Hipparchus was the first to assign values to the brightness of the stars, he attributed magnitude one to the brightest stars and to the fainter ones, a magnitude of six. This system was later revised so that a difference of five magnitudes corresponds to a factor of one hundred in brightness. According to Vogt et al. (2019) [4], the Chinese sources that recorded the stars of the Far East, show a large number of faint stars (magnitude five or less) which were grouped in 283 constellations and added up to between 1.385 and 1.450.

In the last century, Thackeray (1963) [5] reported that the total number of stars visible to the naked eye in the entire sky amounted to an approximate value of 6000 , whose magnitude limit is $\approx 6.6$. Thanks to the help of telescopes, the total number of observable stars reached the figure of more than one billion for a 74-inch telescope, and every time the aperture of a telescope is doubled, the possibility of seeing eight times more stars is increased. Notwithstanding the above, the visualization of the night sky with the naked eye is "contaminated" by the brightness of constellations of artificial satellites Hainaut and Williams (2020) [6], which began to be launched in the second decade of the last century.

At present, Mercier's estimates (2019) [7] propose an age of $13.65 \mathrm{E} 9$ years and a radius $\approx 1.28 \mathrm{E} 26 \mathrm{~m}$ for the universe. However, the expansive condition of the universe is not a matter for discussion and its estimated value assumes the "constant" of Planck's experiment at a speed of $67.4 \pm 0.5 \mathrm{~km} \cdot \mathrm{s}^{-1} \cdot \mathrm{Mpc}^{-1}$, (Netchitailo, 2020) [8] although it has recently been calculated at a higher figure of $73.24 \pm 1.7$ $\mathrm{km} \cdot \mathrm{s}^{-1} \cdot \mathrm{Mpc}^{-1}$ (Riess, et al. 2018) [9], so the radius of the observable universe would be $\approx 4.4 \mathrm{E} 26 \mathrm{~m}$. The oldest known star is HD 140,283 (Methuselah star), is 190 light years distant from the Earth and an age of 14,460 \pm 0.8 million years, has been determined, a figure that does not conflict with the age of the Universe, $13,770 \pm 0.06$ million years, based on the microwave background and Hubble constant (Netchitailo, 2020) [8].

Therefore, this research is based on three assumptions: the first, supported by the existence of a visual convergence plane of the night sky that receives photons of light emitted from distant stars to the Earth, which arrive with a theoretical delay of value $c=299,792,458 \mathrm{~m} \cdot \mathrm{s}^{-1}$ (Hippke, 2018) [10]. Due to this assumption, human vision does not perceive photons at the emission source (stars hundreds of light years distant), but at a near convergence distance after traveling through space. The second, based on the recognition that the observation of the night sky is interfered with by constellations of artificial satellites that orbit the 
Earth, whose distances are linked to their brightness and the brightness of the stars visible to the naked eye. The third, proposes a homogeneous and isotropic flat universe verifiable from distances of the order of 600 million light years (Wuenscheen, 2003) [11]. Thus, the objective of this study is to triangulate the three assumptions and develop mathematical models to propose a valid estimate of the population of stars in the universe.

\section{Methods}

\subsection{Data Collection}

From the Yale Bright Star Catalog (1991) [12], the number of stars visible to the naked eye by apparent magnitude was obtained and from (AAVSO, 2013) [13] the value of the limiting magnitude of naked eye visibility of the stars was verified, for environmental conditions of rural observation. On the other hand, the list of the constellations of the artificial satellites of Hainaut and Williams (2020) [6] was accessed, classified by distance from Earth and the respective brightness.

\subsection{Data Processing}

To build the visual convergence plane, two models were made. The first based on the distances of the satellites and their brightness, correlated to the brightness of the stars (satellite-stellar brightness model). The second is based on the number of light-emitting stars on the surface of a convergence plane (quantity of light model), which according to Traversa (2020) [14] has a two-dimensional value of 4.62E-9 light stars $\mathrm{m}^{-2} \cdot \mathrm{s}^{1}$, which due to the Light travel is interpreted as the probability $(p)$ of finding light sources and not star density. Descriptive statistics techniques of central tendency and dispersion were applied. In parallel, inferential statistics tools (confidence intervals) were used. The regression between the brightness and the height of the satellites was found, which reported the regression coefficients for the equation of best fit between both variables. The apparent distance of the visual convergence plane of the stars was obtained by substitution in the adjustment equation. The average apparent brightness distance was considered for the radius $(r)$ of a circular projection of two-dimensional surface display (S). The radius distance $(r)$ of the first model was compared with the radius distance $(r)$ of the second model. Finally, both models were integrated into a single equation, to find the value $(p)$ and thus be able to determine its magnitude and its behavior as a variable or constant.

\section{Results and Discussion}

\subsection{Visual Convergence Planes}

The descriptive statistics of the brightness of the 9.097 stars, reported that the category with the highest probability is comprised in the interval [6.25 - 6.40]; class in which the majority number of stars is found (Figure 1). 


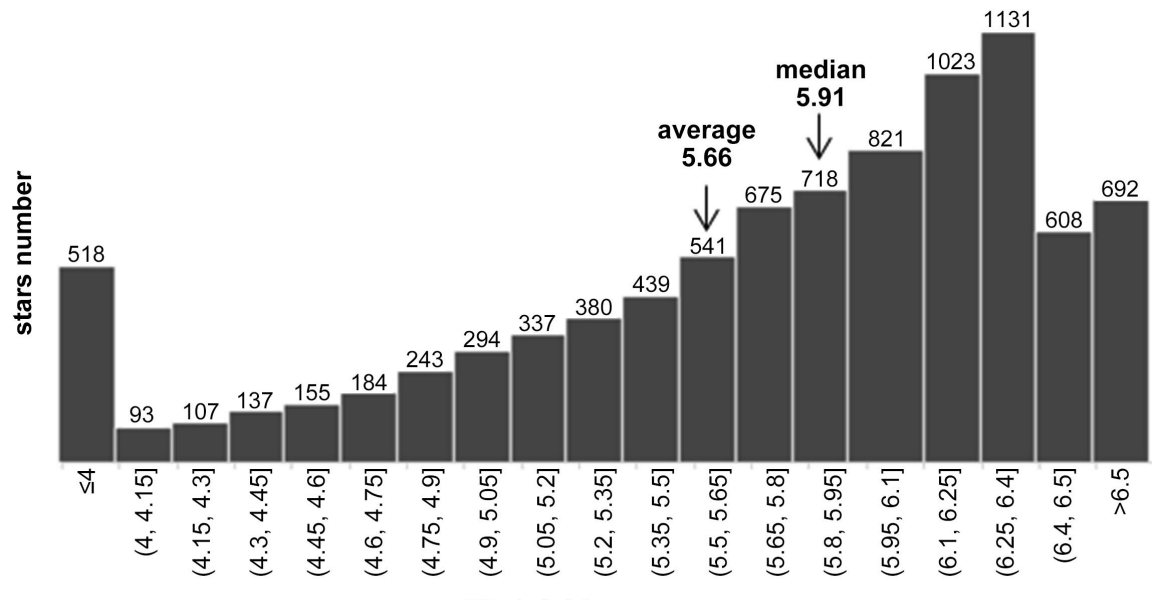

$[\mathrm{X}]$ brightness

Figure 1. Frequency of stars as a function of brightness.

Taking as reference the sample mean of brightness (5.66) and its standard deviation of 0.89; Inferential statistics constructed a confidence interval for the population mean of brightness $(\mu)$, comprised between the values [5.64 - 5.68], for a confidence of $95 \%$ (Equation (1)).

$$
P=\left[\bar{x}-t_{n-1,1-\alpha / 2}(s) / \sqrt{n} \leq \mu \leq \bar{x}+t_{n-1,1-\alpha / 2}(s) / \sqrt{n}\right]=1-\alpha
$$

where: $\bar{x}=$ sample average,

$t_{n-1,1-\alpha / 2}=$ Student's $t$-value $(n ; 95 \%)$,

$s=$ sample standard deviation,

$n=$ sample size.

According to Brekke (2012) [15] with the naked eye, between 5000 and 8000 stars can be observed and according to Collison and Poe (2013) [16], a maximum of 7500 stars can be visible in optimal conditions of darkness and contrast, which would reach up to 9500 according to Müller et al. (2012) [17]. For the Yale Bright Star list, the probability density distribution of the brightness of the 9.097 stars shifts to the right where the median is (5.91) and where the values converge at the limit of vision of the human eye adjusted to the Pogson's logarithmic scale (Fujiwara Yamaoka, 2004) [18].

For the total of 26.816 artificial satellite observations, the approximate mean height was $890 \mathrm{~km}(892)$ and the mean brightness was slightly higher than the naked-eye observation limit $(6.68 \approx 6.7)$. The satellites coincide with the escape zone of $\mathrm{H}+$ and $\mathrm{He}$ from the terrestrial exosphere $(800-900 \mathrm{~km})$ and the location of some telescopes (Noto, 1998) [19] (Table 1).

The 95\% confidence interval for the population mean of the brightness of the satellite constellations $(\mu)$ was between [6.66 - 6.70], also at the limit of human vision; the interval for the average distance from Earth is [888 - 898] $\mathrm{km}$. The best fit regression equation between brightness and distance was of the exponential type (Equation (2)) and Figure 2:

$$
y=a b^{x}
$$

where: $x=$ brightness of the celestial body, 


$$
\begin{aligned}
& y=\text { estimated height }(\mathrm{km}), \\
& a=39.2, \\
& b=1.57 .
\end{aligned}
$$

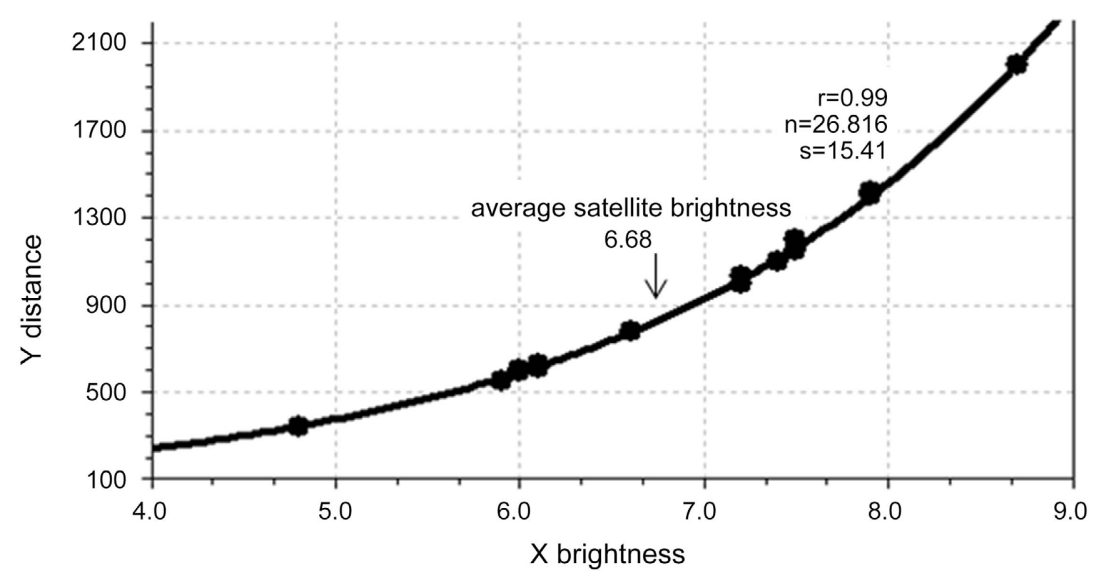

Figure 2. Exponential regression, brightness and distance of artificial satellites or-

\begin{tabular}{|c|c|c|c|c|c|}
\hline Satellites & fi & $\mathrm{X}$ (brightness) & $\begin{array}{c}\text { Y distance } \\
(\mathrm{km})\end{array}$ & $\begin{array}{c}\wedge \text { Y estimated } \\
(\mathrm{km})\end{array}$ & $(\mathrm{fi})^{\star}$ distance \\
\hline Space X Starlink 340 & 7518 & 4.8 & 340 & 344 & $2,556,120$ \\
\hline Space X Starlink 550 & 1600 & 5.9 & 550 & 566 & 880,000 \\
\hline Space X Starlink 1150 & 2800 & 7.5 & 1150 & 1167 & $3,220,000$ \\
\hline One Web & 648 & 7.5 & 1200 & 1167 & 777,600 \\
\hline Amazon Kuiper 590 & 784 & 6 & 590 & 592 & 462,560 \\
\hline Amazon Kuiper 610 & 1296 & 6.1 & 610 & 619 & 790,560 \\
\hline Amazon Kuiper 630 & 1156 & 6.1 & 630 & 619 & 728,280 \\
\hline Sat Revolution & 1024 & 4.9 & 350 & 360 & 358,400 \\
\hline China CASC & 320 & 7.4 & 1100 & 1115 & 352,000 \\
\hline China Lucky Star & 156 & 7.2 & 1000 & 1019 & 156,000 \\
\hline China Commmsat & 800 & 6 & 600 & 592 & 480,000 \\
\hline China Xinwei & 32 & 6 & 600 & 592 & 19,200 \\
\hline India Astro Tech & 600 & 7.9 & 1400 & 1398 & 840,000 \\
\hline Boing & 2956 & 7.2 & 1030 & 1019 & $3,044,680$ \\
\hline LeoSat & 108 & 7.9 & 1423 & 1398 & 153,684 \\
\hline Sammsung & 4700 & 8.7 & 2000 & 2008 & $9,400,000$ \\
\hline Yalini & 135 & 6 & 600 & 592 & 81,000 \\
\hline Telesat LEO & 117 & 7.2 & 1000 & 1019 & 117,000 \\
\hline Iridium & 66 & 7 & 780 & 777 & 51,480 \\
\hline Average & 1411 & 6.7 & 892 & 893 & 912 \\
\hline Standard deviation & 1917 & 1.0 & 424 & 423 & 82 \\
\hline C V \% & 135.9 & 15.6 & 47,5 & 47,4 & 9,0 \\
\hline
\end{tabular}
biting the Earth.

Table 1. Estimated height by brightness of artificial satellites orbiting the Earth.

[Taken and modified from (Hainaut and Williams, 2020) [6]]. 
The deviation (S) of the regression is 15.41 and according to Mallama (2020) [20], the brightness deviation is explained by the uncertainty of the reported magnitudes, the variety of observer measurement techniques, the stars used as reference measurement, the approach to the nearest whole number, the orientation of the satellite and the geographic location of the observer. However, the regression adjustment coefficient $(r)$ was 0.99 .

If the average brightness (X) of the 9.097 stars is included in Equation (2), the apparent distance of the convergence plane is $609 \mathrm{~km}$, its coefficient of variation (\%) is 22.6 and the limits of the confidence interval (95\%) for the population mean $(\mu)$ are [608 - 610] km. To obtain the most distant limit of the visual convergence plane, the detectable brightness in a rural area (6.2) proposed by AAVSO (2013) [13] should be taken; Under these environmental conditions, the regression equation reported a maximum possible distance of $648 \mathrm{~km}$.

According to Traversa (2020) [14], in a universe with a tendency to uniformity and isotropy, it is possible to apply the probability gradient theorem to the delay in the arrival of light photons (delay theorem). Thus, the probability of finding light from a star (light emitting light source) is $2.31 \times 10^{-9}$ in one meter and in one second; for two dimensions it is $(2) \times\left(2.31 \times 10^{-9}\right)$, whose $p$-value $=$ $4.62 \times 10^{-9} \mathrm{~m}^{-2} \cdot \mathrm{s}^{1}$ (Equation (3)).

$$
p=2\left[1-\sqrt[c]{\frac{1}{2}}\right]
$$

where: $p=$ probability of light from a light source $\mathrm{m}^{-2} \cdot \mathrm{s}^{1}$,

$c=3.00 \mathrm{E}+08 \mathrm{~m} \cdot \mathrm{s}^{-1}$ (light delay).

The surface visualized by the human eye receives photons of light from approximately 9097 emission sources (stars) from outer space. If probability is considered as a relative frequency (frequents), it is possible to propose as the number of favorable cases the light of stars observed with the naked eye per second and as the number of total cases to the area of the circle of convergence of the observable plane (Equation (4)).

$$
p=n / N
$$

where $n$ : number of favorable cases; $N$ : number of total cases.

When observing the night sky, the surface of the visual convergence plane in its circular projection is the sample space of the total cases, so by substituting $N$ for the area of the circle, we obtain Equation (5):

$$
A=\frac{n}{p}=\frac{9.097\left(\text { stars } \cdot \mathrm{s}^{1}\right)}{4.62 \mathrm{E}-9\left(\text { ligth stars } \mathrm{m}^{-2} \cdot \mathrm{s}^{1}\right)}
$$

Then, by substituting $A=\pi r^{2}$ in Equation (5), we arrive at Equation (6) (quantity of light model):

$$
r=\sqrt{\frac{n}{\pi p}}
$$

By solving for $r$ (surface radius of the observable convergence circle), the av- 
erage value obtained is $610 \mathrm{~km}$, a figure very close to the average of the previous model $(609 \mathrm{~km})$ that shows the relevance of both propositions (Table 2).

In this way, the satellite stellar brightness model and the amount of light can be integrated into a single model of the visual convergence plane, since the distance to the convergence plane and the radius of the convergence circle are approximate (Figure 3).

The radius of the surface of the observable convergence plane is in an area sensitive to solar radiation (Bauer and Lemmer, 2004) [21] and, according to Bueche (1977) [22], in it the high-energy photons from the Sun break the molecules into ions (ionosphere). Under a homogeneous and isotropic universe (UHI) model (Räsänen, 2009) [23], the density of points of light (stars) per unit area could be an estimator of the probability of light traveling (a figure that should be higher than the previous). For the sample size of the 7000 central observations of starlight's in the magnitude range [4.60 - 6.15], the linear regression of the estimated radius $(\mathrm{m})$ between the satellite brightness model and the quantity of light presented $R^{2}=0.99$; (Figure 4).

From Equation (4), we can solve for $(p)$ as: $n / \pi r^{2}$. It is possible to find the value of $p$ (probability of light $\mathrm{m}^{-2} \cdot \mathrm{s}^{1}$ ) as a function of $n$ (number of stars accumulated per brightness) on the square of the radius of convergence of the circular visual plane multiplied by $\pi$.

Then, using Equation (7), we can now triangulate and substitute $r$ for the distance (height in $\mathrm{m}$ ) from Equation (2) as:

$$
p=\frac{\sum_{i=1}^{i=y} y_{i}}{\pi\left(a b^{x_{i}}\right)^{2}}
$$

where: $p=$ probability of light source $\mathrm{m}^{-2} \cdot \mathrm{s}^{1}$,

$$
\begin{aligned}
& y_{i}=\text { number of stars accumulated to a brightness } i, \\
& x_{i}=\text { brightness of a star } i, \\
& a=39.2, \\
& b=1.57 .
\end{aligned}
$$

The probability distribution of surface light $(p)$ as a function of stellar brightness presents a stable horizontal trend for the circular projection, with an average value equal to $4.62 \mathrm{E}-9$ (starlight $\mathrm{m}^{-2} \cdot \mathrm{s}^{1}$ ) and a reduced coefficient of variation. This is confirmatory evidence that $p=4.62 \mathrm{E}-9\left(\right.$ starlight $\left.\mathrm{m}^{-2} \cdot \mathrm{s}^{1}\right)$ is a universal constant, since it is a sample derived from 5000 astronomical observations (Figure 5).

Table 2. Parameters of the satellite-stars brightness and amount of light models.

\begin{tabular}{ccc}
\hline Parameter & $\begin{array}{c}\text { Satellite-Stars Brightness } \\
\text { radius }(\mathrm{m})\end{array}$ & $\begin{array}{c}\text { Light Quantity } \\
\text { radius }(\mathrm{m})\end{array}$ \\
\hline Average $(\mathrm{m})$ & 609 & 610 \\
Median $(\mathrm{m})$ & 614 & 616 \\
Standard deviation $(\mathrm{m})$ & 68.2 & 82.3 \\
Coefficient of variation $(\%)$ & 22.6 & 32.9 \\
\hline
\end{tabular}




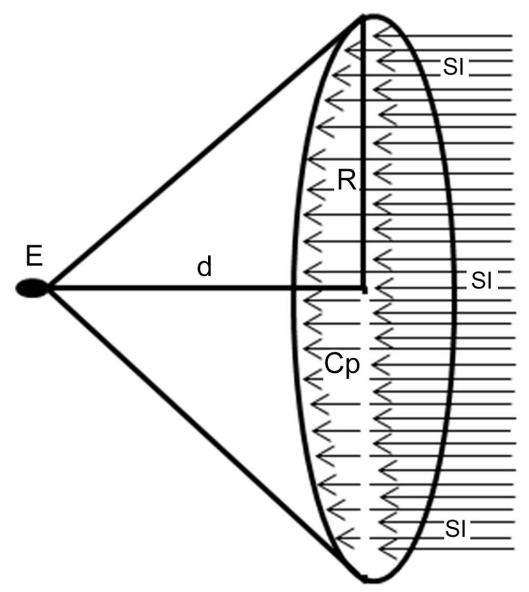

Figure 3. Flat Convergence Model [E = earth; $\mathrm{R}$ = radius of convergence plane; $\mathrm{d}=$ distance to the convergence plane $(\mathrm{Cp}) ; \mathrm{Cp}=$ convergence plane; $\mathrm{Sl}=$ Starlight].

\section{$r(\mathrm{~m})$ brightness satellital}

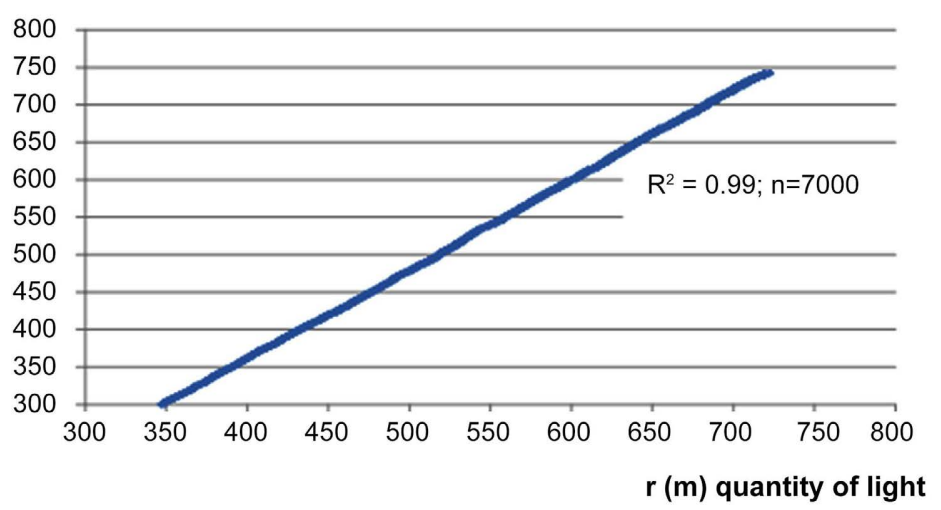

Figure 4. Linear regression, estimated radius (m) between the quantity of light model and the satellite brightness model.

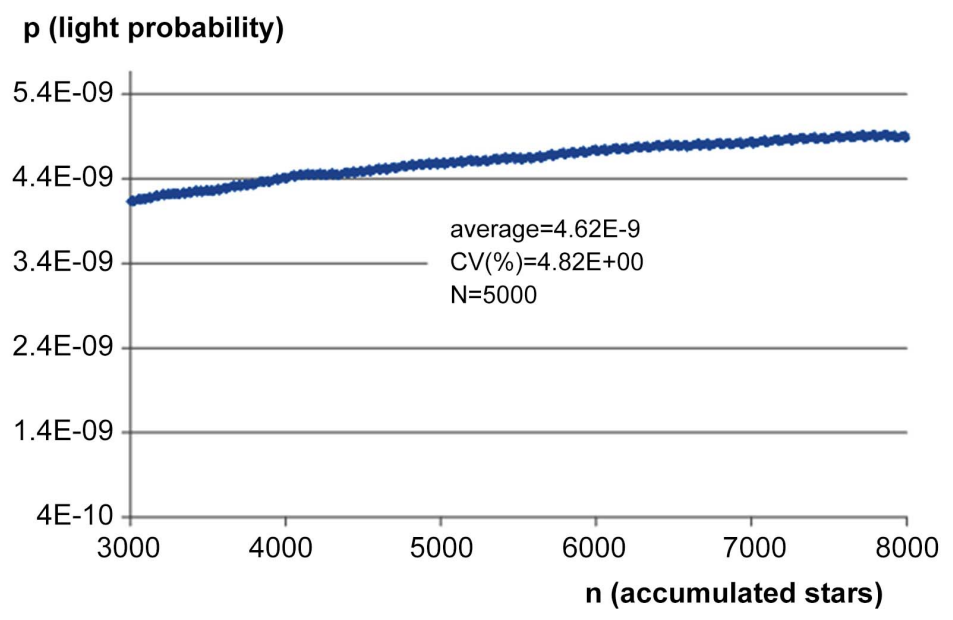

Figure 5. Distribution of the probability of light on the surface $\left(\mathrm{m}^{2}\right)$ as a function of stellar brightness. 


\subsection{Star Estimation for the Universe Model}

The emergence and extinction of stars is framed in a Binomial probability distribution model of presence-absence, with parameters $n$ (sample size) and $p$ (probability of success in each trial). As it is a limiting case, the random variable of the number of successes (stars) can be considered within the Poisson model in an interval of value $\lambda / n$. The mathematical expectation $E(x)=\lambda$ and since $E(x)=$ $n{ }^{\star} p, n$ can be considered as the space-time of the universe taken at the origin (Big Bang). Only solar-like objects are able to shine for billions of years and to be observable until the present universe: thanks to the parameters characterizing our universe (Hujeirat, 2018) [24]. If, according to Mercier (2019) [7], the universe was born from a disturbance that occurred 13.65E9 years ago millions of years (which is equivalent to $4.3 \mathrm{E} 17$ seconds) and moves at a speed c; the current size of the shock wave is $13.65^{\star} 2 \pi$. Under the flat universe model (Shaikh and Bhoyar, 2015) [25] and with a radius of the universe $\approx 1.28 \mathrm{E} 26 \mathrm{~m}$ (Mercier, 2019) [7]; the number of stars in the universe can be obtained as 8 :

$$
E(x)=\lambda=\frac{\left(\frac{R u}{2 \pi}\right)^{2}}{T u} * p
$$

where: $\lambda=$ mathematical expectation of the number of stars in the universe,

$R u=$ radius of the universe $(\mathrm{m})$,

$T u=$ universe time (s),

$p=4.62 \mathrm{E}-9$ (starlight $\left.\mathrm{m}^{-2} \cdot \mathrm{s}^{1}\right)$,

$2 \pi=$ expansive factor of the universe wave.

By substituting for the values of Equation (8), we obtain:

$$
E(x)=\lambda=\frac{\left(\frac{1.28 \mathrm{E} 26}{2 \pi}\right)^{2}}{4.3 \mathrm{E} 17} * 4.62 \mathrm{E}-9 \approx 4.5 \mathrm{E} 24 \text { stars }
$$

For this estimated radius of the observable universe, an approximate value of 4.5E24 stars is obtained, the figure is higher than the estimate of Lazo (2015) [26] (6E22 stars) and similar to the value recently reported by Staff (2019) [27] (1E24 stars) who recognizes that they could be underestimated in quantity. According to (Hujeirat, 2018) [24], in the absence of heavy elements, massive stars must have collapsed into huge stellar black holes, which reside at the centers of observable galaxies. In that sense, equation eight can also be applied to galaxies of known diameter and age. For the Milky Way, the following results are obtained:

$$
E(x)=\lambda=\frac{\left(\frac{5 \mathrm{E} 20}{2 \pi}\right)^{2}}{4.26 \mathrm{E} 17} * 4.62 \mathrm{E}-9 \approx 6.9 \mathrm{E} 13 \text { stars }
$$

\section{Conclusion}

The correlation between the satellite brightness model and the surface light quan- 
tity model showed a high fit. The probability of photon emission [sources = stars], per area is a constant of $4.62 \mathrm{E}-9 \mathrm{~m}^{-2} \cdot \mathrm{s}^{1}$. The value is derived from the Gradient Probability Theorem (TGP). Due to the theorem, in the universe the probability of physical events is not $100 \%$ random or $100 \%$ causal. Randomness explains a maximum of $50 \%$ and causality explains the remaining $50 \%$. Although in theory it is allowed to go to infinity without an event occurring. The TGP shows that a large sample of size (n-1) (not infinite) is enough for an event inevitably occurring with a cumulative probability of value 0.5 . The TGP reconciles mathematics with physics by including speed (delay) in probability of an event. Since light has its speed, a probability is also assigned. The estimate of the total population of stars in the universe is $\approx 4.5 \mathrm{E} 24$.

\section{Conflicts of Interest}

The author declares no conflicts of interest.

\section{References}

[1] Raasveldt, H.C. (1956) Investigación de la visión estereoscópica. Separata de la Revista de la Academia Colombiana de Ciencias. Boletín Geológico, 10, 5-58.

[2] García, F.J., Taylor, M.J. and Kelley, M.C. (1997) Two-Dimensional Spectral Analysis of Mesospheric Airglow Image Data. Applied Optics, 36, 7374-7385. https://doi.org/10.1364/AO.36.007374

[3] Storchi-Bergmann, T., Riffel, R.A., Riffel, R., et al. (2012) Two-Dimensional Mapping of Young Stars in the Inner 180 pc of NGC 1068: Correlation with Molecular Gas Ring and Stellar Kinematics. The Astrophysical Journal, 755, Article No. 87. https://doi.org/10.1088/0004-637X/755/2/87

[4] Vogt, N., Hoffmann, S.M. and Tappert, C. (2019) Sobre las posibilidades de las identificaciones de novas clásicas entre las observaciones históricas de estrellas invitadas del Lejano Oriente. Astronomische Nachrichten, 340, 752-759. https://doi.org/10.1002/asna.201913635

[5] Thackeray, A.D. (1963) The Milky Way. South African Journal of Science, 59, $553-$ 558.

[6] Hainaut, O.R. and Williams, A.P. (2020) Impact of Satellite Constellations on Astronomical Observations with ESO Telescopes in the Visible and Infrared Domains European Southern Observatory. Astronomy \& Astrophysics, 636, 12 p.

[7] Mercier, C. (2019) Calculation of the Mass of the Universe, the Radius of the Universe, the Age of the Universe and the Quantum of Speed. Journal of Modern Physics, 10, 980-1001. https://doi.org/10.4236/jmp.2019.108065

[8] Netchitailo, V.S. (2020) World-Universe Model-Alternative to Big Bang Model. Journal of High Energy Physics, Gravitation and Cosmology, 6, 133-158. https://doi.org/10.4236/jhepgc.2020.61012

[9] Riess, A.G., Casertano, S., Yuan, W., et al. (2018) Milky Way Cepheid Standards for Measuring Cosmic Distances and Application to Gaia DR2: Implications for the Hubble Constant. ArXiv: 1804.10655. https://arxiv.org/abs/1804.10655

[10] Hippke, M. (2018) Interstellar Communication: Short Pulse Duration Limits of Optical SETI. Journal of Astrophysics and Astronomy, 39, Article No. 74.

https://doi.org/10.1007/s12036-018-9565-y 
[11] Wuensche, C.A. (2003) Cosmología. Instituto Nacional de Pesquisas Espaciais.

[12] Yale Bright Star Catalog. https://www.google.com/search?q=star+brigth+catalogue+xlsx\&oq=star+brigth+ca talogue $+x$ ls\&aqs=chrome.1.69i57j33i10i160.17355j0j7 \&sourceid $=$ chrome\&ie $=U T F-8$

[13] AAVSO (2013) Capitulo 3-Haciendo Observaciones. https://www.aavso.org/sites/default/files/publications files/manual/spanish 2013/S Chapter3-2013.pdf

[14] Traversa, I.P.T. (2020) Posibilidad de nueva luz estelar, bajo la teoría del gradiente de probabilidad. Revista Brasileira de Física Tecnológica Aplicada, 7, 1-22. https://doi.org/10.3895/rbfta.v7n1.11705

[15] Brekke, P. (2012) Our Explosive Sun: A Visual Feast of Our Source of Light and Life. Springer, New York.

[16] Collison, F.M. and Poe, K. (2013) "Astronomical Tourism": The Astronomy and Dark Sky Program at Bryce Canyon National Park. Tourism Management Perspectives, 7, 1-15. https://doi.org/10.1016/j.tmp.2013.01.002

[17] Müller, D., Engel, J. and Döllner, J. (2012) Single-Pass Rendering of Day and Night Sky Phenomena. Vision, Modeling, and Visualization, Magdeburg, 12-14 November 2012, 8 p.

[18] Fujiwara, T. and Yamaoka, H. (2004) Magnitude Systems in Old Star Catalogues. ArXiv: Astro-ph/0309322v4. https://arxiv.org/pdf/astro-ph/0309322.pdf

[19] Noto, J. (1998) The Velocity Distributions of Hydrogen and Helium in the Earth's Exosphere. Ph.D. Thesis, Boston University, Boston, 162 p.

[20] Mallama, A. (2020) Starlink Satellite Brightness before VisorSat. ArXiv: 2006.08422. https://arxiv.org/abs/2006.08422

[21] Bauer, S. and Lemmer, H. (2004) Planetary Aeronomy: Atmosphere Environments in Planetary Systems. Springer-Verlag, Berlin.

[22] Bueche, F. (1988) /Ciencias Físicas. Reverté, Barcelona, 432 p.

[23] Räsänen, S. (2009) Light Propagation in Statistically Homogeneous and Isotropic Dust Universes. Journal of Cosmology and Astroparticle Physics, 2009, 11-13. https://doi.org/10.1088/1475-7516/2009/02/011

[24] Hujeirat, A.A. (2018) On the Ultimate Fate of Massive Neutron Stars in an Ever Expanding Universe. Journal of Modern Physics, 9, 51-69. https://doi.org/10.4236/jmp.2018.91004

[25] Shaikh, A.Y. and Bhoyar, S.R. (2015) Plane Symmetric Universe with $\Lambda$ in $f(R, T)$ Gravity. Prespacetime Journal, 6, 1179-1197

[26] Manojlović, L.M. (2015) Photometry-Based Estimation of the Total Number of Stars in the Universe. Applied Optics, 54, 6589-6591. https://doi.org/10.1364/AO.54.006589

[27] ESA (2019) How Many Stars Are There in the Universe? European Space Agency. 